\title{
Recognition of Cross Browser Inconsistency by crawling and comparing extracted attributes
}

\author{
C.P.Patidar*, Meena Sharma**, Varsha Sharda*** \\ *(Department of Information Technology, IET, DAVV, Indore \\ ** (Department of Computer science, IET, DAVV, Indore \\ *** (Department of Computer science, Medicaps, Indore
}

\begin{abstract}
The advancement over web innovation is distinguishment for web applications that amplifies inconsistencies between different web browsers. These incompatibilities supplement cross browser incompatibilities that contain modified look at evolving browsers for a specific web application. For a percentage cases, Cross-Browser Inconsistencies (XBIs) comprises for satisfactory difference, while these might actually prevent clients starting with gaining entrance to and only a web application's purpose for different situations. Therefore, testing procedure of a web provision must a chance to be performed efficiently looking into different browsers with accomplish consistency. Available techniques and tools oblige a significant manual exertion on remember such issues; also furnish restricted help to settling those reason for the issues. In this paper, we recommend a technique to identifying cross-browser issues without human obstruction.
\end{abstract}

Keywords: Browser, Cross Browser, Web Application

\section{INTRODUCTION}

Currently, web applications would develop from web frameworks or sites dependent upon a client server model. When a client issues a request to the server through a web browser then the server side components get invoked. These interchanges transform solicitations of the server, and the server responds should such solicitations with updates of the current web page, programmed in html (HyperText mark up language), alternately XML (extensible Mark-up Language), furthermore with different related resources, for example, style data to CSS (Cascading style Sheets), client-side code (e.g., JavaScript), images, etc. Subsequently, these assets are used to figure and render an updated web page in the web program.

A web program may be a programming provision to retrieving, delivering and furthermore traversing majority of the data assets on the Web. Eventually perusing a uniform resource locator (URL), a web page, picture and data asset will be perceived. Those programs get into contact with the web server and also necessities for majority of the data. Those web servers receive the majority of the data and show it on the machine. The major issue joins with working on those web applications through separate web browsers are identified with web browser incompatibility. Likewise, web applications are constantly utilized to a large number of exercises over each zone. A few divergences for presentation of components or substance of a webbased application around diverse browsers may be known as Cross-Browser Inconsistency. When a customer run a web application on many browsers, then some web application demonstrates different behaviors and as a result introduces Cross-Browser Inconsistencies (XBIs) [1]. If cross browser inconsistencies are not being legitimately tried for the testing phase, subsequently it could negatively impact the knowledge of the client of the web application. Consequently, a key component is with distinguishing those cross browser inconsistencies is an extreme worry to organizations reliant on such applications for the growth of their business [2].

It takes a look that; website will be created utilizing particular case program as opposed to numerous browsers. Trying over a go for browsers will picture issues that designer might make ignorant for. Accordingly, we performed a deliberate investigation looking into different real-world web applications. This investigation facilitates us to discover a classification for XBIs that helps in characterizing our system. We set up three major varieties of XBIs:

1. Structural XBIs: It happens when one or more components of a particular web page are misaligned looking into different browsers [3].

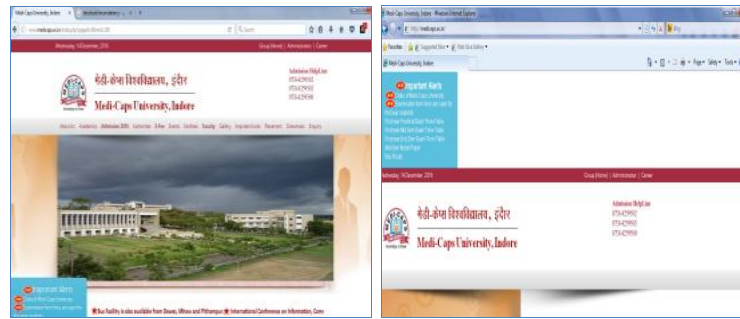

Fig.1: Example of Structural XBI 
2. Content XBIs: This sort of XBI may be exhibit in the substance of a particular web page. Such debate might take place, the graphical angle of a web page element, alternately those text based quality about an element, is separate over two browsers. It can again classified as visual-content and text-content XBIs [3].

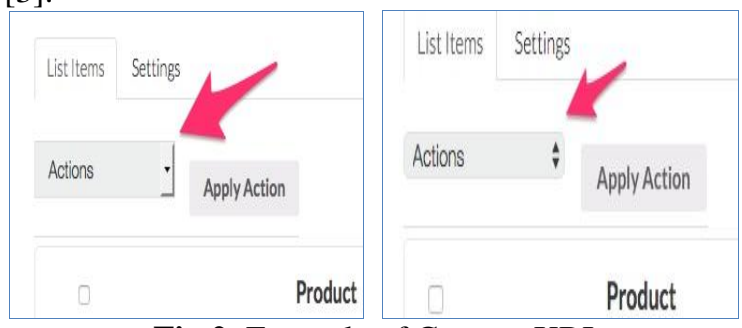

Fig.2: Example of Content XBI

3. Behavioral XBIs: These sorts of XBIs involve contrasts in the conduct technique about distinct widgets on a web page. A sample of such a XBI might be a connection that redirects to in turn web page inside one browser and a completely distinctive page or no redirection at all, in another browser[3].For example we run https://www.zomato.com/indore/delivery on two different browsers i.e., internet Explorer and Google Chrome.

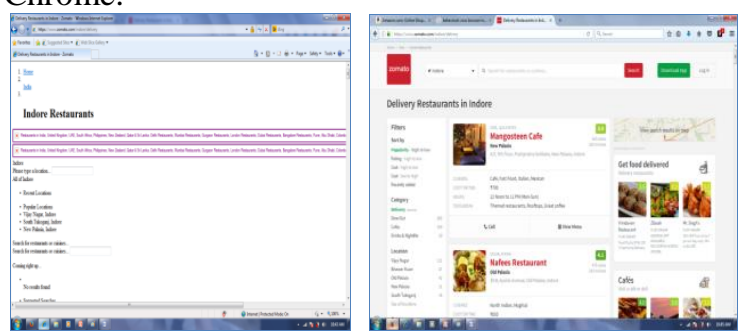

Fig.3: Example of Behavioral XBI

In addition, the internet of web application has gently become one of the vital medium of the business. The software faults in web applications have potentially lead to the failure or the underperformance of the line. Key quality attributes for web applications include reliability, availability, interoperability and security apart from ensuring the functional \& usability aspects [4].Web browser compatibility testing is technical and puzzlingsomething you must give your web designer to manage. That issue may be that if your website is not well-suited with the different browsers available, it will interfere with your benefits of the business notoriety [5].

On deliver the drawbacks from existing techniques, we suggested a strategy that mixes a profound spot for correlation strategies and orchestrates them to apply each procedure of the classification about XBIs that it may be best matched with recognize. Our proficiency will be a computerized, defined and a broad approach to XBI identification. The key offerings of this work are:

- A new technique and tool for detecting both visual and structural XBIs in web applications.

- An innovative, powerful technique to detect visual XBIs.

- An assessment of this proficiency on various real-world web applications that demonstrates its effectiveness in detecting different kinds of inconsistencies XBIs.

The rest of the paper is structured as follows. Section I contain introduction of cross browser inconsistency along with web application, Section II contain background and related work done in area of cross browser inconsistency of a web application, Section III describes problem definition of our research work .Section IV explains our proposed solution to identify cross browser inconsistency with flow chart, Section V contain expected outcomes of our proposed methodology and Section VI concludes research work with future directions.

\section{BACKGROUND AND RELATED STUDY}

To accomplishing our objective for identifying cross-browser issues automatically, our methodology needs to beat some tests. Firstly, any manual review to identify such issues may be exorbitant in provisions of assets for example, period and effort. For this reason, the method should be entirely computerized and ought to work without requiring any manual exertion starting with developers. Secondly, the technique should investigate the structural information of the web page over various browsers and extract all words and content of the web page. This can be done with the help of a web crawler. Before performing any comparison, our technique needs to extract different attributes from crawler statistics when same web page has been executed on different browsers.

Our methodology comprises of attribute extraction algorithm such as vertex connectivity or neighborhood similarity for extracting attributes. To identify cross browser inconsistency over different browsers, comparison of the web page components is a big challenge. Web applications habitually need variable components for example, promotions and created substance (egg. time, date and so on.) which need aid diverse over various solicitations. If these components need aid not mistreated, that strategy might think about the progressions crosswise over browsers along with bringing about false positives in the outcomes. Thus, the method obliges finding and also taking off out such components throughout examination.

At last the challenge is due to the inbuilt safety parameters in web browsers that pose a 
specialized challenge for the strategy to clearly take out all required information for comparison.

The recent work on recognizing XBIs has recommended techniques that focus only on some aspects of a web application's execution, and are well suitable for specific types of XBIs. For instance, a tool that uses computer vision to detect XBIs is WebDiff [6], whereas a creature that uses graph isomorphism along with text comparison to find XBIs is Cross T [7].These tools provide just halfway and loose answers for those XBI identification issue. An additional approach tests the trouble for cross-browser similarity of web applications concerning illustration of a practical consistency check from web application conducts a technique over different web browsers for a robotized result. This approach comprises of analyzing the provided web application automatically under various different browser platforms and recording the activities as a finite-state machine and comparing the generated models for similarity on a pair wise basis and illuminating any examined dissimilarities [8].

Further, a tool for naturally recognizing XBIs over web applications, without requiring exertion from the designer need to have been furnished[9].This tool can work with any web application that runs on desktop browsers. This model captures screen and then compares the graph generated by crawler by the graph isomorphism checking method. Also, it identifies different types of inconsistencies in a web application. It also generates easy to understand and actionable reports for the developer, as a result allowing them to deal with XBIs more proficiently.

Survey of web applications for different types of inconsistencies:-

We have manually tested the websites on following browsers: Google Chrome, Mozilla Firefox and Internet Explorer.

Table 1

\begin{tabular}{|c|c|c|c|c|c|}
\hline \multirow[t]{3}{*}{ S.NO } & \multirow[t]{3}{*}{ URL } & \multicolumn{4}{|c|}{ TYPE OF INCONSISTENCY } \\
\hline & & \multirow{2}{*}{$\begin{array}{l}\text { Structu } \\
\text { ral }\end{array}$} & \multicolumn{2}{|c|}{ Content } & \multirow{2}{*}{$\begin{array}{l}\text { Behavio } \\
\text { ral }\end{array}$} \\
\hline & & & Text & Visual & \\
\hline 1 & http://www.yahoo.coc & & Yes & yes & \\
\hline 2 & http://www.medicaps.ac.in & Yes & & & \\
\hline 3 & http://www.ebay.in & Yes & & & \\
\hline 4 & $\begin{array}{l}\text { http://www.sports.dauniv.ac.in/gallery } \\
\text { /photo-gallery.php }\end{array}$ & & & & Yes \\
\hline 5 & http://www.indoreinstitute.com/iimr/ & Yes & & & \\
\hline 6 & http://www.nitdelhi.ac.in/ & & & & Yes \\
\hline 7 & http://www.gingerhotels.com / & Yes & & & \\
\hline 8 & http://www.indore.chokhidhani.com/ & & & yes & \\
\hline 9 & http://www.holidayiq.com/ & & & yes & \\
\hline 10 & $\begin{array}{l}\text { https://enigma- } \\
\text { medicaps.herokuapp.com/ }\end{array}$ & & Yes & & \\
\hline 11 & https:/www.zomato.com/indore/ & & Yes & yes & \\
\hline 12 & http://craftswilla.com/ & Yes & & & \\
\hline 13 & http://craftswilla.com/ & & Yes & & \\
\hline
\end{tabular}

Findings of the survey:

Behavioral XBIs - $15 \%$

Structural XBIs - 39\%

Content based XBIs $-46 \%$

\section{PROBLEM DEFINITION}

The objective of a good web design is to provide the one and the same appearance of the web application viewed from any web browser. As every web page is made up of varieties of content and customized data and the execution of a web page in a variety of contexts has been getting influenced by it. Alike to other parameters of performance evaluation, different parts of the web page also bears upon the browser compatibility feature of internet site either directly or indirectly. In addition, different technologies produce the compatibility issue. As a result, for the period of the design stage of the websites must be tested thoroughly for its compatibility at different browsing environments. The parameters that can affect the cross browser inconsistency of a web application have been discussed in Section II

\section{Description}

\section{PROPOSED SOLUTION}

To identify cross browser inconsistencies, we propose a methodology [11] to detect XBI. Figure 1 depicts an outline of our proposed XBI detection methodology that takes URL of any web application as an input. Now the provided URL will be executed on selected browser from browser list. Identified inconsistencies will be the expected result of the proposed methodology, if any. This methodology is composed of five different components, each of which is described below.

\section{(i) Web Crawler}

Web crawler is software for downloading pages from the Web automatically. It is also called web spider or web robot. Web crawling is an important method for collecting data on, and keeping up with, the rapidly expanding Internet [12]. It crawls different websites and produces graphs for a specific website. For crawling a website, we take some input from user for e.g. Option of traversing (BFS, DFS), max page size, crawl delay etc. On the basis of these parameters, it generates a graph for corresponding website.

\section{(ii) Attribute Extractor}

After generating graph by web crawler as mentioned above, some specific attributes are get extracted by attribute extractor. Attributes are the parameters those affect cross browser compatibility. Attribute extractor component extracts the attributes such as HTML tags, CSS, Response time, IP address of a web page, text type, text size, image type, These 
parameters are get extracted for each browser and store the values for a browser of these parameters in the database. To extract attribute from graph, an algorithm known as graph clustering will be used [13].

\section{(iii) Comparator}

This component compares the attributes extracted by attribute extractor. If value of any parameter is somehow different for two different browsers then there can be some sort of inconsistencies but if value of any parameter is same for multiple browsers, then there are no cross browser inconsistencies present. To find text content XBIs, this component performs textual analysis of comparable components. To detect image content XBIs, it compares image parameters of the corresponding elements on the web page.

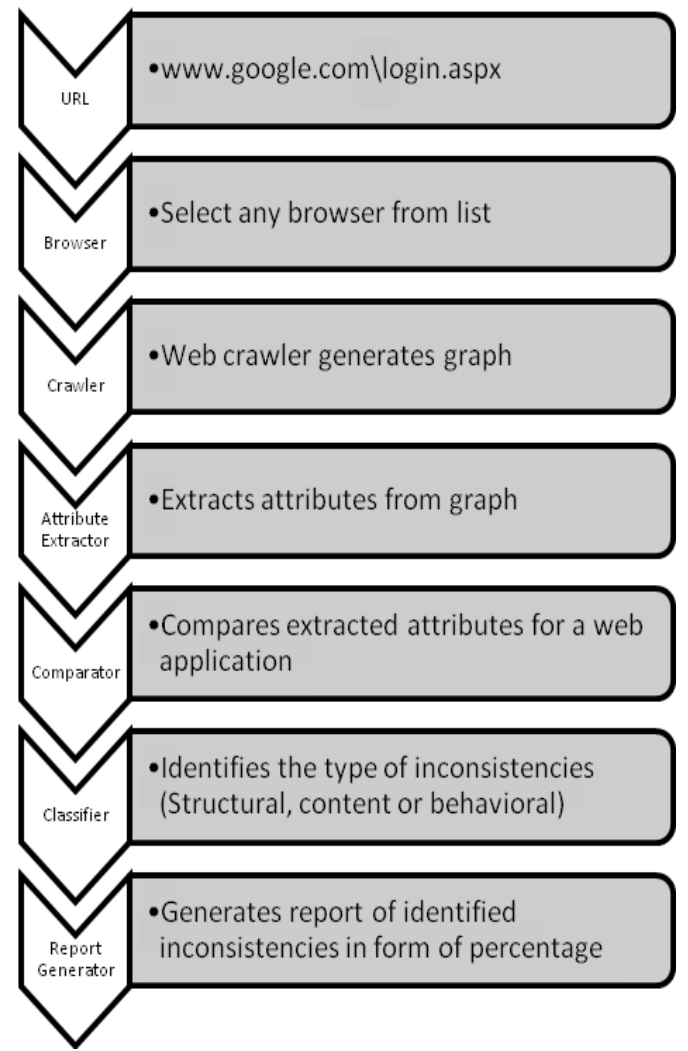

Fig. 4: Model for Cross Browser Inconsistency

\section{(iv) Classifier}

This component classifies the type of inconsistencies on the basis of specific parameters. It identifies that whether identified inconsistency is structural, content or behavioral inconsistency. For e.g. if a parameter image: height is different for two browsers then it is content inconsistency.

\section{(v) Report Generator}

This module generates a report that tabulates the set of identified XBIs written in HTML. It generates the report of inconsistencies in form of percentage.

\section{EXPECTED OUTCOMES}

It has been noted that, when a web application is performed on multiple browsers, then expected outcome of our model is suggested to distinguish three major cases of inconsistencies, if any. This proposed model also generates reports of incompatibilities. Therefore, finding of XBIs can be a fast technique as compared to other available tools. These are illustrated as below:

- We found that, structural XBIs are the most general class of XBIs, happening in $39 \%$ of the subjects with XBIs.

- We ascertain that these content XBIs occurred in $20 \%$ and $26 \%$ of the sites with XBIs respectively.

- We determine that behavioral XBIs occurred in $15 \%$ of the web applications with XBIs.

Thus, we can conclude a reason that behavioral XBIs have an impact on the purpose of individual components, bringing about broken route the middle of distinctive screens. On the other hand, Structural and content XBIs include dissimilarities in the structure or content of a specific web page.

\section{V.CONCLUSION}

To develop a web application, cross browser inconsistencies would be an extreme issue. This severe problem must be taken carefully in testing phase. Existing research techniques focus on only some specific part from XBIs and can report a huge number about false positives and negatives. So, to manage these limitations, we exhibited our suggested model for identification of XBI. To achieve this job this paper presents the general idea of the projected methodology, procedure and expected output. For addition, it also generates straightforward reports for the developer that includes the detected cross browser inconsistencies for a particular web application in form of percentage, consequently permitting them to manage XBIs in a greater amount successfully. This is a difficult complexity, because the application will essentially appear unlike in multiple browsers, but it should provide the identical or related functionality at the minimum. After the text edit has been completed, the paper is ready for the template. Duplicate the template file by using the Save As command, and use the naming convention prescribed by your conference for the name of your paper. In this newly created file, highlight all of the contents and import your prepared text file. You are now ready to style your paper; use the scroll down window on the left of the MS Word Formatting toolbar. 


\section{REFERENCES}

[1] C.P.Patidar and Meena Sharma,"An automated approach for cross browser inconsistency (XBI) detection", Ninth annual ACM India conference organized by ACM India, Oct 21-23, 2016.

[2] Nepal Barskar, C.P.Patidar and Meena Sharma, "Analysis and Identification of Cross Browser Inconsistency Issues in Web Application using Automation Testing", International Journal of Computer Science and Information Technology \& Security (IJCSITS), ISSN: 2249-9555Vol.6, No3, May-June 2016.

[3] Nepal Barskar and C.P. Patidar, “A Survey on Cross Browser Inconsistencies in Web Application”, International Journal of Computer Applications (0975 - 8887) Volume 137 - No.4, March 2016.

[4] "Web Testing", www.mindlance.com, testing@mindlance.com.

[5] Ochin and Jugnu Gaur, "Cross Browser Incompatibility: Reasons and Solutions", International Journal of Software Engineering \& Applications (IJSEA), Vol.2, No.3, July 2011.

[6] Shauvik Roy Choudhary, Husain Versee and Alessandro Orso, "WEBDIFF: Automated Identification of Crossbrowser Issues in Web Applications", $26^{\text {th }}$ IEEE International Conference on Software Maintenance in Timisoara Romania, 978-1-4244-8628-1/10, 2010.

[7] Ali Mesbah and Mukul R. Prasad,“ Automated Cross-Browser Compatibility Testing”, ICSE '11,Waikiki, Honolulu, HI, USA ,ACM 978-1-4503-0445-0/11/05, May 21-28, 2011 .

[8] Shauvik Roy Choudhary, Mukul R. Prasad and Alessandro Orso, "CROSSCHECK: Combining Crawling and Differencing to Better Detect Cross-browser Incompatibilities in Web Applications", 2012.

[9] Shauvik Roy Choudhary, Mukul R. Prasad and Alessandro Orso, “X-PERT: A Web Application Testing Tool for Cross-Browser Inconsistency Detection”, ISSTA’14,San Jose, CA, USA,Copyright 2014 ACM 978-1-4503-26452/14/07,July 21-25, 2014.

[10] Shauvik Roy Choudhary,'Detecting Cross-browser Issues in Web Applications”, ICSE '11, Waikiki, Honolulu, HI, USA, ACM 978-1-4503-0445-0/11/05, May 21-28, 2011.

[11] C.P.Patidar, Meena Sharma and Varsha Sharda, "Detection of Cross Browser Inconsistency by Comparing Extracted Attributes", International Journal of Scientific Research in Computer Science and Engineering, Vol.5, Issue.1, pp.1-6, 2017.

[12] Trupti V. Udapure, Ravindra D. Kale and Rajesh C. Dharmik,"Study of web crawler and its Different types", IOSR Journal of Computer Engineering (IOSR-JCE) e-ISSN: 2278-0661, p- ISSN: 2278-8727Volume 16, Issue 1, Ver. VI (Feb. 2014), PP 01-05

[13] Yang Zhou, Hong Cheng and Jeffrey Xu Yu ,"Graph Clustering Based on Structural/Attribute Similarities", in ACM VLDB'09, Lyon France, August 24-28, 2009

\footnotetext{
International Journal of Engineering Research and Applications (IJERA) is UGC approved Journal with S1. No. 4525, Journal no. 47088.

C.P .Patidar. "Recognition of Cross Browser Inconsistency by crawling and comparing extracted attributes ." International Journal of Engineering Research and Applications (IJERA) 7.7 (2017): 57-61.
} 